\title{
Xenotransplantation of embryonic pig pancreas for treatment of diabetes mellitus in non-human primates ${ }^{*}$
}

\author{
Marc R. Hammerman
}

George M. O'Brien Center, Department of Medicine, Washington University School of Medicine, St. Louis, USA

Email: mhammerm@dom.wustl.edu

Received 7 March 2013; revised 8 April 2013; accepted 8 May 2013

Copyright (C) 2013 Marc R. Hammerman. This is an open access article distributed under the Creative Commons Attribution License, which permits unrestricted use, distribution, and reproduction in any medium, provided the original work is properly cited.

\begin{abstract}
Transplantation therapy for diabetes in humans is limited by the low availability of human donor whole pancreas or islets. Outcomes are complicated by immunosuppressive drug toxicity. Xenotransplantation is a strategy to overcome supply problems. Implantation of tissue obtained early during embryogenesis is a way to reduce transplant immunogenicity. Pig insulin is biologically active in humans. In that regard the pig is an appropriate xenogeneic organ donor. Insulin-producing cells originating from embryonic pig pancreas obtained very early following pancreatic primordium formation [embryonic day 28 (E28)] engraft long-term in rhesus macaques. Endocrine cells originating from embryonic pig pancreas transplanted in host mesentery migrate to mesenteric lymph nodes, engraft, differentiate and improve glucose tolerance in rhesus macaques without the need for immune suppression. Transplantation of embryonic pig pancreas is a novel approach towards beta cell replacement therapy that could be applicable to humans.
\end{abstract}

Keywords: Beta Cell; Diabetes Mellitus; Non-Human Primates; Transplantation; Xenotransplantation

\section{INTRODUCTION}

There are approximately 1000 pancreas and islet transplants performed per year in the USA, the diabetic population in which exceeds 20 million individuals [1]. Transplantation is rarely used for type 2 diabetes which is almost 20 times more common than type 1 disease. When performed, whole pancreas or islet transplantation requires use of potent immunosuppressive medications that have significant complications [2]. Pig insulin works well in humans. For that reason, the pig could be a

\footnotetext{
$z^{*}$ Supported by George M. O’Brien Center DK079333.
}

physiologically suitable beta cell donor [2]. The severity of humoral rejection effectively precludes the use of pigs, as whole pancreas donors. However, because they are vascularized by the host post-transplantation, islets are not subject to humoral rejection [3]. Unfortunately, porcine islets are rejected by the cellular immune system within days of transplantation in non-immune suppressed non-human primates. Experience with non-transgenic or transgenic pig to primate islet or neonatal islet transplantation in immune suppressed non-human primates shows that sustained insulin independence can be achieved, but only through the use of agents that are not approved for human use or that result in a high level of morbidity and mortality $[3,4]$. Thus, the need for host immune suppression is a barrier for pig-to-human islet xenotransplantation.

The use of embryonic tissue for transplantation offers theoretical advantages relative to transplantation of either pluripotent embryonic stem (ES) cells, or of fully differentiated (adult) organs [reviewed in 4]: 1) Unlike ES cells, organ primordia differentiate along defined organ-committed lines. There is no requirement to steer differentiation and no risk of teratoma formation; 2) The growth potential of cells within embryonic organs is enhanced relative to those in terminally-differentiated organs; 3) The cellular immune response to transplanted primordia obtained early during embryogenesis is attenuated relative to that directed against adult organs; 4) Early organ primordia are avascular. The ability of cellular primordia to attract a host vasculature renders them less susceptible to humoral rejection than are adult organs with donor blood vessels transplanted across a discordant xenogeneic barrier; and 5) Organ primordia differentiate selectively. In the case of embryonic pancreas, exocrine pancreatic tissue does not differentiate following transplantation, obviating complications that can result from exocrine components such as the enzymatic autodigestion of host tissues.

Approximately a decade ago, we observed that embryonic pig kidney, transplanted across a wide xenogeneic 
barrier into immune-suppressed rodent (inbred mouse or rat) mesentery undergoes differentiation into an anatomically precise renal organ at the transplant site $[5,6]$. When we attempted the same approach using embryonic pig pancreas rather than kidney, we discovered using two inbred rat strains as hosts [Lewis rats rendered diabetic using streptozotocin (STZ) [6-9], a model for type 1 diabetes in humans or Zucker Diabetic Fatty (ZDF) rats [10], a model for type 2 diabetes] that unlike kidney, embryonic pancreas does not differentiate in situ into an anatomically precise pancreas. Rather: 1) pancreas differentiates selectively in that only endocrine cells survive; 2) endocrine cells differentiate and migrate into host mesenteric lymph nodes from where they release insulin in a glucoseregulated manner, the host serving as its own in vivo bioreactor; and 3) host immune suppression, required for engraftment of transplanted embryonic pig kidney into mice or rats is not required for engraftment of early-stage embryonic pancreas.

Strategies for xenotransplantation in humans are modeled immunologically using old world monkeys or baboons as hosts. This is because the non-human primates share uniquely with humans a complex and redundant immune system that includes the capacity for humoral rejection of vascularized implants originating from pigs, a frequently used donor-species, outbred diversity and MHC expression patterns known to present barriers to long-term graft survival [3]. Employing protocols developed for embryonic-pig to STZ-diabetic Lewis rat embryonic pancreas transplantation and recapitulated in ZDF rat hosts, we showed that embryonic pig pancreas engrafts, and improves glucose tolerance in STZ-diabetic rhesus macaques without the need for immune suppression [11,12]. Here we review the adaptation of pig-into-diabetic rat transplantation technology to a pig-into-diabetic non-human primate model.

\section{TRANSPLANTATION OF EMBRYONIC PANCREAS}

\subsection{Transplantation of Embryonic Pig Pancreas in Diabetic Rhesus Macaques Is Followed by Engraftment of Pig Endocrine Cells in Mesenteric Lymph Nodes}

The developmental stage of donor pig embryos from which primordia are obtained impacts on the host immune response. We showed E35 pig pancreatic primordia are rejected in Lewis rats following transplantation employing conditions under which E28 pig pancreatic primordia engraft [6]. While we have not determined whether E30-E34 pig pancreas has a fate similar to organs obtained on E35, we always use E28 pig pancreas as a source for transplantation because it engrafts predictably. It follows that the importance of obtaining ac- curately and precisely-timed pregnant Yorkshire sows from which to obtain E28 embryos is critical to the success of experiments. An E28 pig pancreatic primordiumwith separate dorsal pancreas (dp) and ventral pancreas (vp) components is shown as an inset (Figure 1(A)) in Figure 1. It is approximately $1 \mathrm{~mm}$ in diameter (much smaller than primordia obtained from older embryos). and its dissection from pig embryos requires a good deal of skill. Our ability to employ E28 embryonic pig pancreas for transplantation is dependent on obtaining pregnant sows that are exactly 28 days pregnant from which to obtain embryos. There is no pancreas in embryos obtained from sows that are 26 days pregnant. Thus there is little or no margin for error.

Following incubation for 45 minutes in vitro of dissected E28 pig pancreatic primordia with selected growth factors and cytokines, we implant individual dorsal and ventral components, within a tunnel dissected between tissue planes of mesentery. Figure 1(B) shows the mesentery of a STZ-diabetic rhesus macaque at the time of transplantation. A primordium between sheets of mesentery is delineated (arrowhead). Accumulation of fat and proliferation of lymphatic vessels are observed in mesentery within 6 weeks post-transplantation in non-human primates [11,12]. Figure 2 shows photomicrographs originating from a mesenteric lymph node of a rhesus macaque transplanted previously with E28 pig pancreatic primodia in mesentery. Sections in Figures 2(A) and (C) are stained with an anti-insulin antibody. Sections in Figures 2(B) and (D) are incubated with control serum. Individual cells that stain positive (red) are present in medullary sinus (arrow A). The cells are polygonal, consistent with a beta cell identity (Figure 2(C) arrow). No positive-staining cells are found in sections incubated with control serum (Figures 2(B) and (D)). No insulinpositive cells are present in mesenteric lymph nodes of

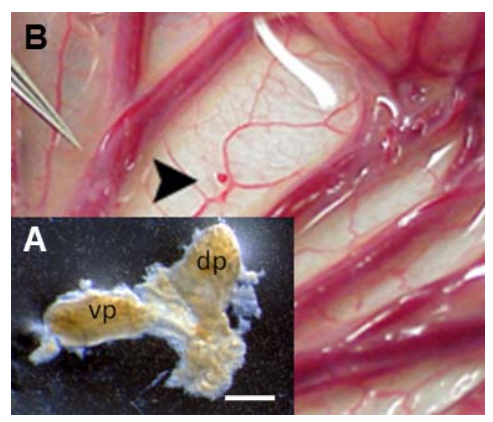

Figure 1. (A) Photograph of a pancreatic primordium freshly dissected from an E28 pig embryo; (B) A pancreatic primordium implanted between sheets of mesentery in a rhesus macaque. dp, dorsal pancreas; vp, ventral pancreas. Scale bar 10 um (A). Reproduced with permission [11]. 
non-transplanted rhesus macaques [11]. Engraftment of pig tissue in the mesenteric lymph nodes is documented using in-situ hybridization for porcine proinsulin mRNA. Cells expressing porcine mRNA stain with use of an antisense probe to porcine proinsulin mRNA (Figure 2(E)), but not a sense probe (Figure 2(F)). The presence of porcine tissue engrafted in lymph nodes of primates is confirmed by detecting porcine proinsulin mRNA using reverse-transcriptase polymerase chain reaction (RT$\mathrm{RCR}$ ), porcine $\mathrm{X}$ chromosomes using fluorescence insitu hybridization, and cells with insulin granules in lymph node tissue using electron microscopy $[11,12]$.

\subsection{Improvement of Glucose Intolerance Following Transplantation of E28 Pig Pancreatic Primordia and Demonstration of Porcine Insulin in Circulation}

Figure 3 shows the results of intravenous glucose tolerance testing in rhesus macaques prior to administration of STZ (Pre-STZ) and in the same animals following induction of STZ-diabetes (Post-STZ) and after transplantation of E28 pig pancreatic primordia (Post TX).

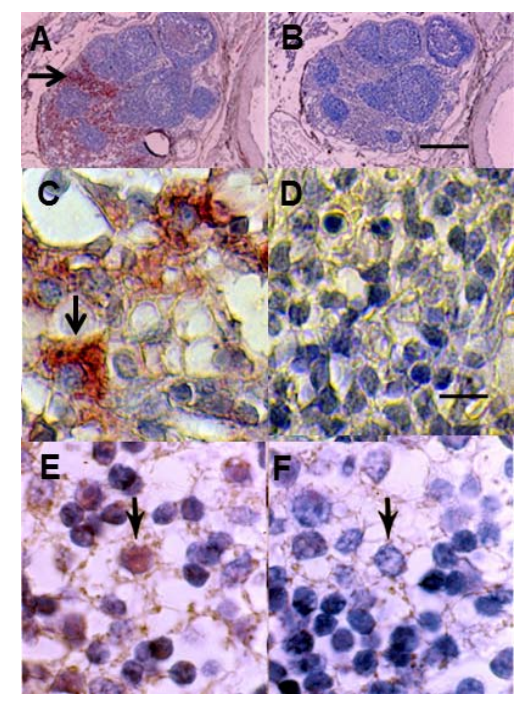

Figure 2. Photomicrographs of mesenteric lymph nodes from a STZ-diabetic diabetic rhesus macaque posttransplantation of E28 pig pancreatic primordia. Sections A and C are stained with an anti-insulin antibody. Sections $\mathrm{B}$ and $\mathrm{D}$ are stained using a control serum. Arrow delineates medullary sinus (A) Arrow delineates polygonal cell; (C) In situ hybridization was performed using antisense (E) or sense probes (F). Arrows delineate a cell in consecutive sections to which the antisense probe binds (E). Scale bars 80 um (A) \& (B); 10 um (C)-(F). Reproduced with permission [11].

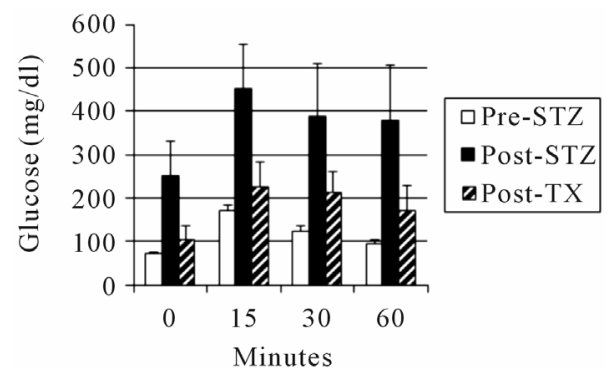

Figure 3. Intravenous glucose tolerance in rhesus macaques $(n=3)$. Glucose in peripheral venous blood was measured prior to intravenous infusion of dextrose (Time 0 ) and at several times after infusion, in animals prior to administration of STZ (Pre-STZ); 5 days following administration of STZ (Post STZ); or following transplantation of $20-40$ E28 pig pancreatic primordia in mesentery (Post-TX). Data are shown as mean \pm SE. Reproduced with permission [12].

Glucose tolerance is nearly normalized in diabetic rhesus macaques following transplantation of E28 pig pancreatic primordia. No antibody can distinguish porcine from primate (or human) insulin. Accordingly we distinguished between the two in the circulation of rhesus macaques using sequential high performance liquid chromatography, immunoaffinity chromatography and mass spectrometry [11]. No rhesus macaque insulin $(<0.1$ $\mathrm{ng} / \mathrm{ml}$ ) can be detected post-transplantation of E28 pig pancreatic primordia in diabetic rhesus macaques. Rather porcine insulin is detectable in plasma (Figure 4).

\subsection{Summary and Conclusions}

The studies reviewed above represent one of two independent decade-long efforts (ours based at Washington University $[11,12]$ and the other at the Weizmann Institute of Science, Rehovot Israel $[13,14])$ to adapt xenotransplantation of embryonic pig pancreas as a novel therapy for diabetes mellitus in humans. Hecht et al. [13] (Weizmann Institute) applied findings generated in mice (Tchorsh-Yutsis et al.) [14] to a non-human primate model. The mouse studies had demonstrated the curative potential of such implants in diabetic mice under relatively tolerable immune suppression. A working hypothesis, suggesting that embryonic pig pancreas transplantation would not be adversely affected by a humoral response was based on 2 observations: 1) hyperacute and acute rejection are mainly mediated by complement activation in blood vessels, mediated by preformed anti-pig antibodies recognizing carbohydrates expressed on endothelial cells; and 2) data from the mouse studies showed that the embryonic pancreas implants predominantly induce host-type vasculature to support their growth and development in the recipient, resulting in an 


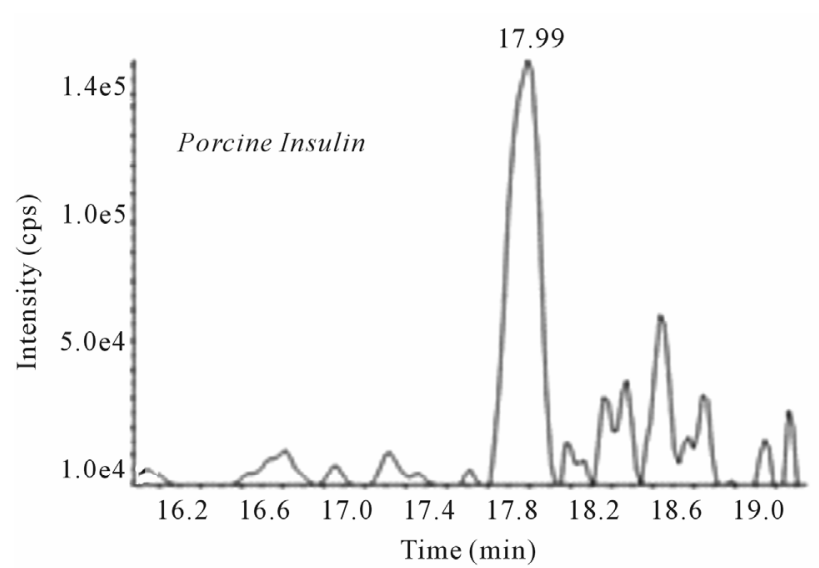

(A)

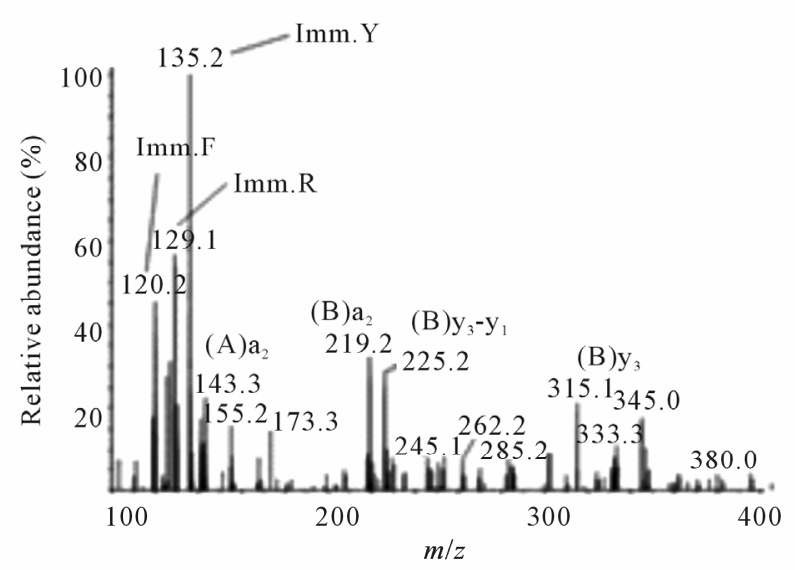

(B)

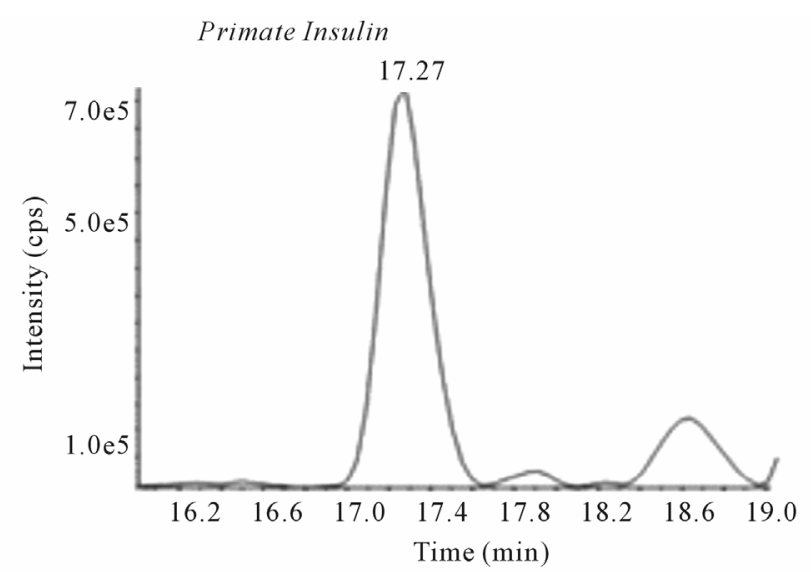

(C)

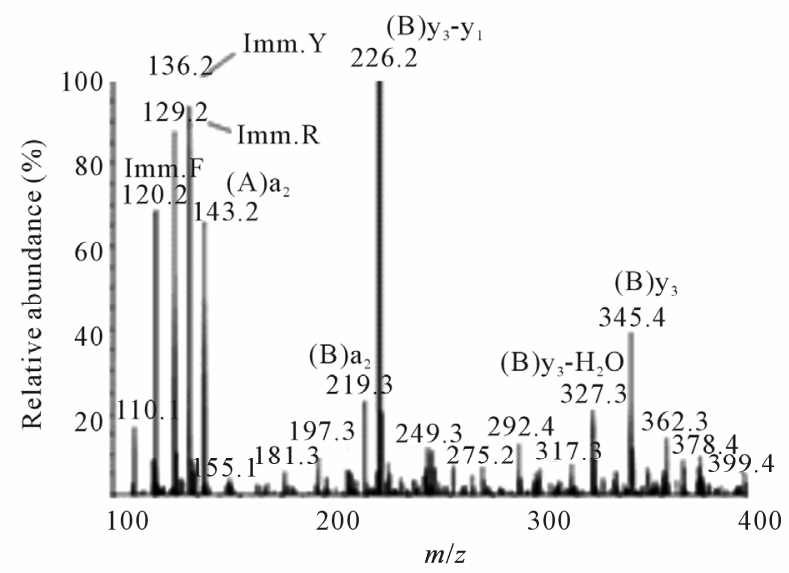

(D)

Figure 4. Chromatogram (A) and product ion mass spectrum (B) of porcine insulin (precursor ion $[\mathrm{M}+5 \mathrm{H}]^{5+} \mathrm{m} / \mathrm{z} 1156.3$ ) extracted from $2 \mathrm{ml}$ plasma obtained 5 minutes after IV glucose administration to STZ-diabetic transplanted rhesus macaque 2 . The retention time and diagnostic product ions derived from the five-fold charged precursor ion unambiguously identify porcine insulin. Chromatogram (C) and product ion mass spectrum (D) of human/macaque insulin (precursor ion $[\mathrm{M}+5 \mathrm{H}]^{5+} \mathrm{m} / \mathrm{z} 1162.3$ ) extracted from $1 \mathrm{ml}$ of non-diabetic non-transplanted rhesus macaque plasma. The retention time and diagnostic product ions derived from the five-fold charged precursor ion unambiguously identify human/macaque insulin. Reproduced with permission [11].

organ comprised mainly of porcine epithelial cells and host endothelium. It was found that that porcine E42 embryonic pancreatic tissue can correct hyperglycemia in diabetic Cynomolgous monkeys under a tolerable immune suppression protocol and was concluded that long-term proliferative capacity of these grafts, their ability to induce revascularization by host endothelium, and their reduced immunogenicity, suggest that porcine embryonic xenotransplantation could offer an attractive replacement therapy for diabetes $[13,14]$.

Host immune suppression was required for successful engraftment of embryonic pig pancreas in rodents [14] or non-human primates [13] in the studies of TchorshYutsis et al. and Hecht et al. It is likely that one or more factors in the methodology we employ different from that used by these investigators is critical for engraftment without an immune suppression requirement. We have speculated regarding what the factors might be [4]. First, as noted above, the developmental stage of donor pig embryos from which primordia are obtained impacts on the host immune response. While we have no experience transplanting E42 pig pancreatic primordia in rats, the preferred stage for studies described by Tchorsh-Yutsis et al. and Hecht et al., we would expect them to reject in the absence of host immune-suppression based on our experience with E35 pancreatic primordia. Second, it is likely that that the incubation of embryonic pancreas with one or more growth factors and cytokines we employ prior to transplantation alters the host immune response. Tchorsh-Yutsis et al. Hecht et al. do not employ such agents. Third, the transplantation site and technique probably impact on the host immune response. We interpose pancreatic primordia between sheets of mesentery (Figure 1(B)). Tchorsh-Yutsis et al and Hecht et al., transplant into pockets of omentum and secure using suture. 
The potential for use of the mesenteric transplant site to enable dissemination of cells along a lymphatic distribution and engraftment in mesenteric lymph nodes was confirmed recently in fumarylacetoacetate mice $\left(\mathrm{Fah}^{-} \Gamma\right.$ ), a model for the human liver disease tyrosinemia type I (reviewed in [4]). Intra peritoneal transplantation of liver cells from congenic wild-type mice resulted in correction of metabolic abnormalities and long-term survival of the animals that otherwise would have died. Transplantation of liver cells into the peritoneal cavity allowed the hepatocytes to migrate into the lymphatic system, enter mesenteric lymph nodes and expand under homeostatic mechanisms driven by the liver. When ectopic liver tissue reached the required balance for hepatic function, proliferation ceased resulting in 20 to 40 "hepatized" mesenteric lymph nodes that represented $70 \%$ of the original liver mass. The authors speculate that the highly vascularized nature of the lymph nodes supports the efficient engraftment and massive expansion of ectopic tissue and can be compared to a well-designed in vivo bioreactor [15]. Little is known about the factors that regulate the differentiation, migration and immunogenicity of cells transplanted within mesentery. Delineation of such factors could enable optimization of engraftment and expansion of endocrine cells originating from E28 pig pancreatic primordia distributed similarly via the lymphatic route.

Whereas rats the glucose tolerance of which is normalized following transplantation of 5 - 8 E28 pig pancreatic primordia are rendered insulin independent for the remainder of their lives, macaques transplanted with 20 - 40 primordia remain dependent on exogenous insulin (albeit as doses lower than required prior to transplantation) to maintain euglycemia [11]. One explanation for the differential success between rats and macaques is that macaques weigh 20 times as much as rats. A STZdiabetic rat can be rendered normoglycemic lifelong with no exogenous insulin requirement by transplantation of 5 - 8 pig pancreatic primordia. Extrapolating, it would take 100 - 200 primordia to render a diabetic macaque independent of exogenous insulin. A second explanation is proposed by Graham and Schuurman in their recent review of the usefulness and limitations of the diabetic macaque model in evaluating long-term survival of porcine islets [16]. The authors propose that there are three main contributors to the mild hyperglycemia that is common in xenografted diabetic non-human primates: 1) species incompatibilitiesunderlying a lower insulin secretion rate by the porcine graft; 2) the set point in insulinsecretion that is at higher blood glucose levels in pigs than in monkeys; and 3) a marginal mass of engrafted islets. The authors speculate the mild hyperglycemia observed in transplanted diabetic primates might not be of high relevance when considering the use of porcine islet transplantation in human diabetics. This is because humans and pigs have normal fasting blood glucose values in a similar range while in primates, the values are much lower. Additionally, the authors suggest, if the intrinsic insulin-producing capacity of porcine islets is lower than that of human islets, the effective dose of porcine islets may need to need to be increased. A similar argument could apply to the "effective dose" of porcine pancreatic primordia, in which case more than 100 200 may be required to render a diabetic macaque independent of exogenous insulin. Alternatively, unlike the case for mature islets, beta cell precursors in transplanted embryonic pancreas have the potential to differentiate into a mass of mature insulin-producing cells sufficient to render a diabetic host euglycemic. A single native embryonic pancreas does so in every non-diabetic pig, primate or human. Future efforts are appropriately directed to engineering transplanted pig pancreatic primordia towards more robust differentiation within the in vivo bioreactor that is the diabetic host.

\section{REFERENCES}

[1] McCullough, K.P., Keith, D.S., Meyer, K.H., Stock, P.G., Brayman, K.L. and Leichtman, A.B. (2009) Kidney and pancreas transplantation in the United States 1998-2007. Access for patients with diabetes and end stage renal disease. American Journal of Transplantation, 9, 894-906. doi:10.1111/j.1600-6143.2009.02566.x

[2] Bottino, R. and Trucco, M. (2005) Multifaceted therapeutic approaches for a multigenic disease. Diabetes, 54, S79-S86. doi:10.2337/diabetes.54.suppl 2.S79

[3] Hering, B.J. and Walawalkar, N. (2009) Pig-to-nonhuman primate islet xenotransplantation. Transplant Immunology, 21, 81-86. doi:10.1016/j.trim.2009.05.001

[4] Hammerman, M.R. (2012) Development of a novel xenotransplantation strategy for treatment of diabetes mellitus in rat hosts and translation to non-human primates. Organogenesis, 8, 41-48. doi:10.4161/org.20930

[5] Rogers, S.A., Talcott, M. and Hammerman, M.R. (2003) Transplantation of pig metanephroi. ASAIO Journal, 49, 48-52. doi:10.1097/00002480-200301000-00008

[6] Rogers, S.A., Liapis, H. and Hammerman, M.R. (2005) Normalization of glucose post-transplantation of pig pancreatic anlagen into non-immunosuppressed diabetic rats depends on obtaining anlagen prior to embryonic day 35 . Transplant Immunology, 14, 67-75. doi:10.1016/j.trim.2005.02.004

[7] Rogers, S.A., Chen, F., Talcott, M. and Hammerman, M.R. (2004) Islet cell engraftment and control of diabetes in rats following transplantation of pig pancreatic analgen. American Journal of Physiology, 286, E502-E509. doi:10.1152/ajpendo.00445.2003

[8] Rogers, S.A. and Hammerman, M.R. (2008) Normalization of glucose post-transplantation into diabetic rats of 
pig pancreatic primordia preserved in vitro. Organogenesis, 4, 48-51. doi:10.4161/org. 5747

[9] Rogers, S.A. Mohanakumar, T, Liapis, H and Hammerman M.R. (2010) Engraftment of cells from porcine islets of Langerhans and normalization of glucose tolerance following transplantation of pig pancreatic primordia in non-immune suppressed diabetic rats. American Journal of Pathology, 177, 854-864. doi:10.2353/ajpath.2010.091193

[10] Rogers, S.A., Chen, F., Talcott, M., Liapis, H. and Hammerman M.R. (2006) Glucose tolerance normalization following transplantation of pig pancreatic primordia into non-immunosuppressed diabetic ZDF rats. Transplant Immunology, 16, 176-184. doi:10.1016/j.trim.2006.08.007

[11] Rogers, S.A., Chen, F., Talcott, M.R., Faulkner, C., Thomas, J.M., Thevis, M. and Hammerman M.R. (2007) Long-term engraftment following transplantation of pig pancreatic primordia into non-immunosuppressed diabetic rhesus macaques. Xenotransplantation, 14, 591-602. doi:10.1111/j.1399-3089.2007.00429.x

[12] Rogers, S.A., Tripathi, P., Mohanakumar, T., Liapis, H., Chen F., Talcott, M.R, et al. (2011) Engraftment of cells from porcine islets of Langerhans following transplanta- tion of pig pancreatic primordia in non-immune suppressed diabetic rhesus macaques. Organogenesis, 7, 154-162. doi:10.4161/org.7.3.16522

[13] Hecht, G., Eventov-Friedman, S., Rosen, C., Shezen, E., Tchorsh, D., Aronovich, A., et al. (2009) Embryonic pig pancreatic tissue for the treatment of diabetes in a nonhuman primate model. Proceedings of the National Academy of Sciences of the United States of America, 106, 8659-8664. doi:10.1073/pnas.0812253106

[14] Tchorsh-Yutsis, D., Hecht, G., Aronovich, A., Shezen, E., Klionsky, Y., Rosen, C., et al. (2009) Pig embryonic pancreatic tissue as a source for transplantation in diabetes: Transient treatment with anti-LFA1, anti-CD48 and FTY720 enables long term graft maintenance in mice with only mild ongoing immune suppression. Diabetes, 58, 1585-1594. doi: $10.2337 / \mathrm{db} 09-0112$

[15] Hoppo, T., Komori, J., Manohar, R., Stolz, D.B. and Lagasse, E. (2011) Rescue of lethal hepatic failure by hepatized lymph nodes in mice. Gastroenterology, $\mathbf{1 4 0}$, 565-666. doi:10.1053/j.gastro.2010.11.006

[16] Graham, M.L. and Schuurman, H-J. (2013) The usefulness and limitations of the diabetic macaque model in evaluating long-term porcine islet xenograft survival. Xenotransplantation, 20, 5-17. doi:10.1111/xen.12012 